03

\title{
Влияние неравновесных приэлектродных слоев на структуру ЭГД течений в трехионной модели диэлектрической жидкости
}

\author{
(C) Ю.К. Стишков, Д.В. Богданов \\ Санкт-Петербургский государственный университет, фризический фракультет, \\ 198504 Санкт-Петербург, Петергоф, Россия \\ e-mail: y.stishkov@spbu.ru
}

(Поступило в Редакцию 11 мая 2016 г. В окончательной редакции 31 августа 2016 г.)

\begin{abstract}
Электрогидродинамические течения представляют собой самопроизвольное течение жидкости в межэлектродном промежутке под влиянием сильного электрического поля. Наибольшее количество экспериментальных данных по исследованию поля скоростей ЭГД течений получено для электродов „провод над плоскостью“. Для этой системы течение можно считать двумерным. Приведены результаты компьютерного моделирования полной системы уравнений электрогидродинамики в трехионной модели диэлектрической жидкости. На основе результатов компьютерного моделирования ЭГД течений в жидкостях различной низковольтной проводимости для системы электродов „провод над плоскостью“ проведен анализ структуры неравновесных диссоционно-рекомбинационных слоев и их влияния на структуру ЭГД течений.
\end{abstract}

DOI: $10.21883 / J T F .2017 .08 .44719 .1882$

\section{Введение}

Экспериментальные исследования показали, что в хорошо очищенных жидких диэлектриках ЭГД течения крайне неустойчивы и слабоинтенсивны [1]. При введении в жидкость электронно-акцепторных примесей интенсивность течений возрастает. Однако с увеличением концентрации примесей собственная проводимость жидкости возрастает, а эффект возрастания интенсивности ЭГД течений замедляется, а затем начинает уменьшаться [1]. В ряде работ увеличение интенсивности ЭГД течений при введении примесей объясняется увеличением интенсивности инжекции [2,3]. Однако объемный заряд, обусловленный инжекцией в жидкость с повышенной собственной проводимостью, ослабевает в связи с рекомбинацией инжектируемого заряда в приэлектродных неравновесных рекомбинационных слоях [4-7], следовательно, ослабевает и интенсивность ЭГД течений. В представляемой работе этот эффект рассмотрен на материалах компьютерного моделирования в рамках трехионной модели диэлектрических жидкостей. Моделирование проведено в пакете COMSOL Multiphysics для системы электродов „провод над плоскостью“, расположенной в кювете из диэлектрического материала. В качестве рабочей жидкости выбрана жидкость со следующими свойствами: диэлектрическая проницаемость 2.2 , плотность $800 \mathrm{~kg} / \mathrm{m}^{3}$, динамическая вязкость $0.01 \mathrm{~Pa} \cdot \mathrm{s}$, в жидкости имеются положительные и отрицательные ионы с подвижностью $8.5 \cdot 10^{-9} \mathrm{~m}^{2} /(\mathrm{V} \cdot \mathrm{s})$, образованные за счет диссоциации примесных молекул, и третий сорт положительных ионов с подвижностью $4.25 \cdot 10^{-9} \mathrm{~m}^{2} /(\mathrm{V} \cdot \mathrm{s})$, образованных из молекул примесей в процессе инжекции. Радиус проволочного электрода $0.25 \mathrm{~mm}$, за время максвелловской релаксации напряжение увеличивалось на $100 \mathrm{~V}$. В работе [5] показано, что в жидких диэлектриках, являющихся очень слабыми электролитами, прохождение электрического тока сопровождается нарушением равновесия между процессами диссоциации и рекомбинации ионов, в результате которого у поверхности электродов возникают так называемые неравновесные приэлектродные диссоционно-рекомбинационные слои. Показано, что в гидростатическом режиме для системы плоских электродов эти слои описываются безразмерным параметром $P_{0}=L^{2} \sigma_{0} / b \psi_{0} \varepsilon \varepsilon_{0}$, равным отношению времени максвелловской релаксации к времени дрейфа иона через межэлектродный промежуток. В отсутствие инжекции у электродов возникают слои, заряженные противоположно электроду, а их относительная толщина характеризуется величиной $1 / P_{0}$ : если $1 / P_{0}<1$, то размеры слоев также меньше длины межэлектродного промежутка. При наличии инжекции структура слоев зависит также и от величины безразмерного параметpa $k$, равного отношению плотности тока инжекции на поверхности проволочного электрода к плотности тока проводимости. При значениях $k<1$ инжектированные ионы рекомбинируют в тонком приэлектродном слое с ионами проводимости и не проникают в объем жидкости. При $k=1$ неравновесные слои не возникают, так как плотность тока инжекции равна плотности тока низковольтной проводимости. При $k>1$ инжектируемые ионы эффективно проникают в объем только при малых значениях безразмерного параметра $P_{0}$, а в противном случае (при $P_{0} \gg 1$ ) инжектированные ионы быстро рекомбинируют, образуя тонкие приэлектродные ионизационно-рекомбинационные слои. В настоящей работе на основе результатов компьютерного моделирования ЭГД течений в жидкостях различной низковольтной проводимости в системе электродов „провод над плоскостью“ проводится анализ структуры неравновесных слоев и их влияния на ЭГД течения. 


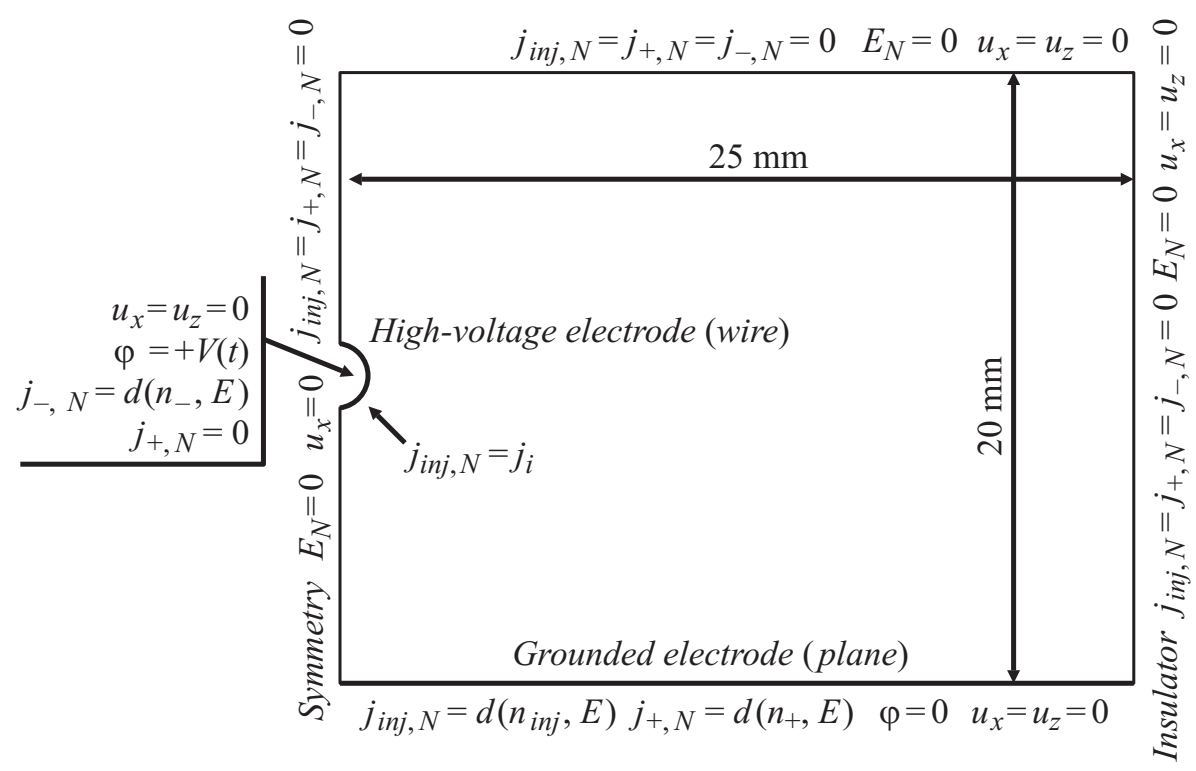

Рис. 1. Геометрия модели и граничные условия.

\section{Система ЭГД уравнений}

$$
\begin{gathered}
\Delta \varphi=-\frac{\rho}{\varepsilon \varepsilon_{0}}, \\
\frac{\partial c_{1}}{\partial t}+\operatorname{div}\left(-c_{1} b_{1,2} z_{1} \nabla \varphi-D_{1} \nabla c_{1}\right) \\
+\mathbf{u} \nabla c_{1}=\alpha_{d}-\alpha_{r 1} c_{1} c_{2}, \\
\frac{\partial c_{2}}{\partial t}+\operatorname{div}\left(-c_{2} b_{1,2} z_{2} \nabla \varphi-D_{2} \nabla c_{2}\right) \\
+\mathbf{u} \nabla c_{2}=\alpha_{d}-\alpha_{r 1} c_{1} c_{2}-\alpha_{r 2} c_{2} c_{3}, \\
\frac{\partial c_{3}}{\partial t}+\operatorname{div}\left(-c_{3} b_{3} z_{3} \nabla \varphi-D_{3} \nabla c_{3}\right) \\
+\mathbf{u} \nabla c_{3}=-\alpha_{r 2} c_{2} c_{3}, \\
\rho=e\left(z_{1} c_{1}+z_{2} c_{2}+z_{3} c_{3}\right), \\
\gamma \frac{\partial \mathbf{u}}{\partial t}+\gamma(\mathbf{u}, \nabla) \mathbf{u}=-\nabla P+\eta \Delta \mathbf{u}-\rho \nabla \varphi, \\
\operatorname{div}(\mathbf{u})=0,
\end{gathered}
$$

где $\varphi$ - электрический потенциал, $\gamma-$ плотность жидкости, и - скорость жидкости, $P$ - давление, $\eta-$ динамическая вязкость, $t-$ время, $\rho-$ объемная плотность заряда, $c-$ концентрация ионов, $b-$ подвижность ионов, $D$ - коэффициент диффузии ионов, $z_{i}$ - валентность ионов, $e-$ элементарный электрический заряд, $\alpha_{d}-$ коэффициент диссоциации, $\alpha_{r}-$ коэффициент рекомбинации, $F-$ константа Фарадея, $\varepsilon$ - относительная диэлектрическая проницаемость и $\varepsilon_{0}$ - диэлектрическая постоянная, $k_{B}-$ постоянная Больцмана, $T-$ температура, $\sigma_{0}-$ низковольтная проводимость жидкости, индекс $i$ указывает на сорт ионов. $\alpha_{d}=\sigma_{0}^{2} / \varepsilon \varepsilon_{0} 2 b_{1,2} e, D_{i}=\frac{k_{B} T}{e} b_{i}$.
В уравнении Навье-Стокса (6) и неразрывности для несжимаемой вязкой жидкости (7) рассчитывается гидродинамика. Массовая сила $f=-\rho \nabla \varphi$ в правой части (6) - это сила Кулона, обобщенная на случай пространственного распределения заряда $\rho$. Потенциал находится из уравнения Пуассона (1), которое учитывает поле, создаваемое распределением заряда $\rho(5)$. Концентрации положительных и отрицательных ионов рассчитываются с помощью двух уравнений Нернста-Планка (2), (3).

В случае учета трех сортов ионов (два типа ионов получаются в результате диссоциации молекул примеси и третий - инжектированные) к системе уравнений добавляется еще одно уравнение Нернста-Планка (4), а в уравнение (3) добавляется член, отвечающий за рекомбинацию отрицательных ионов с ионами третьего сорта: $-\alpha_{r 2} c_{2} c_{3}$. Здесь $\alpha_{r 1}-$ коэффициент рекомбинации отрицательных диссоциированных ионов с положительными диссоциированными ионами, $\alpha_{r 2}-$ коэффициент рекомбинации отрицательных диссоциированных ионов проводимости с положительными инжектированными ионами, $b_{1,2}$ - подвижность диссоциированных ионов, $b_{3}-$ подвижность инжектированных ионов. $\alpha_{r 1}=2 b_{1,2} e / \varepsilon \varepsilon_{0}, \alpha_{r 2}=\left(b_{2}+b_{3}\right) e / \varepsilon \varepsilon_{0}$. В правых частях уравнений Нернста-Планка (2)-(4) стоит „функция источника“, описывающая появление ионов первых двух типов в результате диссоциации молекул и гибель ионов в результате рекомбинации положительных диссоциированных ионов с отрицательными и положительных инжектированных ионов с отрицательными диссоциированными ионами.

На рис. 1 представлены геометрия модели и граничные условия. В процессе моделирования использовался метод линейного повышения напряжения с малой ско- 


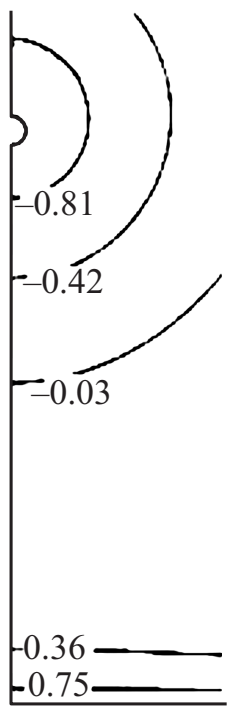

$1.5 \mathrm{kV}$

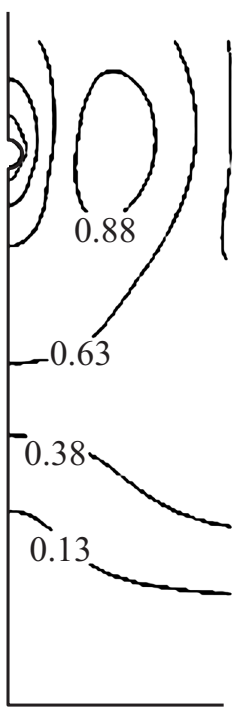

$1.5 \mathrm{kV}$
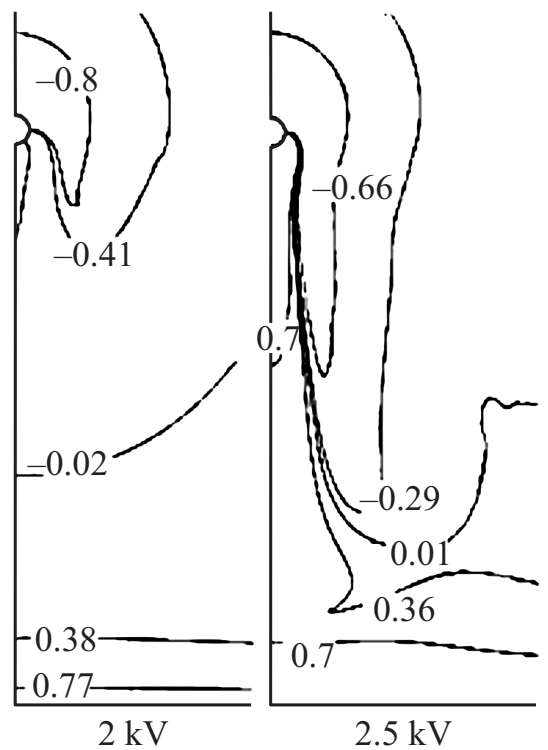

$2.5 \mathrm{kV}$

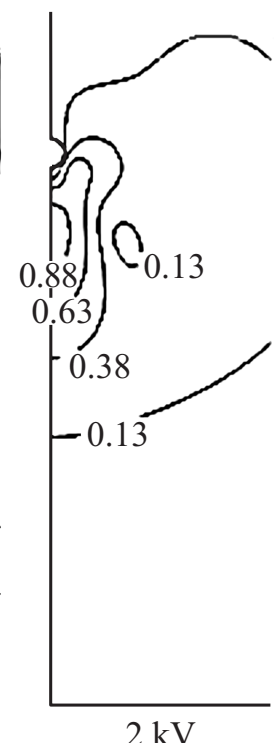

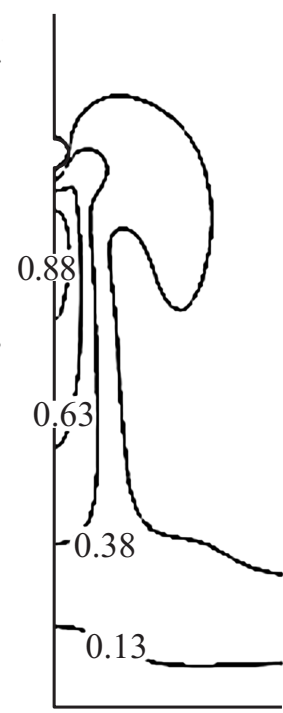

$2.5 \mathrm{kV}$

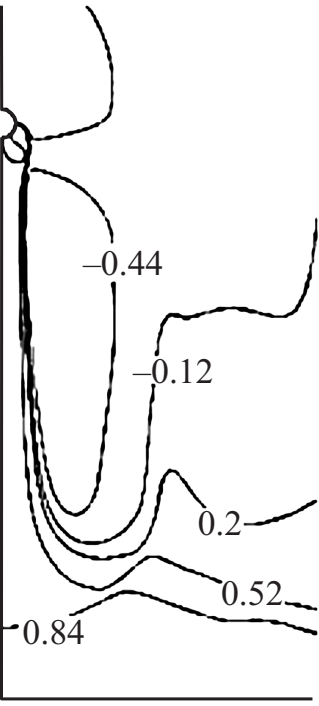

$10 \mathrm{kV}$

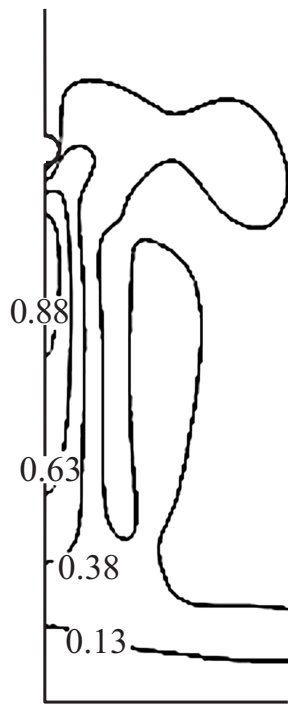

$10 \mathrm{kV}$

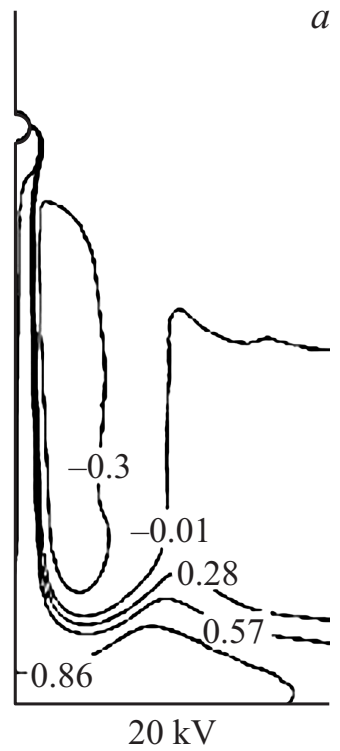

$h$

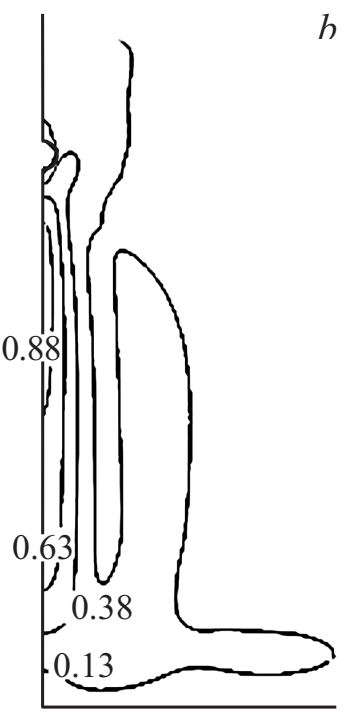

$20 \mathrm{kV}$

Рис. 2. $a$ - распределения объемного заряда в относительных единицах относительных единицах при различных напряжениях в жидкости № 1. $b$ - распределения скорости ЭГД течений в относительных единицах при различных напряжениях в жидкости № 1.

ростью в диапазоне от 0.1 до $20 \mathrm{kV}$. За время максвелловской релаксации напряжение увеличивалось не более чем на $100 \mathrm{~V}$, таким образом, в результате моделирования получена картина установившихся ЭГД течений в указанном диапазоне напряжений.

\section{Результаты моделирования для жидкости с проводимостью $10 \mathrm{pS} / \mathrm{m}$}

На рис. 2 приведены распределения объемного заряда и скоростей ЭГД течений при напряжениях 1.5, 2, 2.5, 10 , 15 и $20 \mathrm{kV}$. В диапазоне малых напряжений $(U<2 \mathrm{kV})$ безразмерный токовый параметр меньше единицы, поэтому у проволочного и плоского электродов формируются неравновесные рекомбинационные слои объем- ного заряда противоположного с электродом знака [5]. У проволочного электрода слой отрицательного заряда имеет кольцевую структуру, у плоского - плоскую. Напряженность электрического поля у проволочного электрода выше, чем у плоского. Соответственно этому величина безразмерного параметра $P_{0}$ у электродов различна. Характерная средняя толщина безразмерного приэлектродного слоя в межэлектродном промежутке, рассчитанная по формуле $1 / P_{0}$, порядка 1 , т. е. размеры неравновесного приэлектродного слоя по порядку соответствуют межэлектродному промежутку.

На графиках рис. 2 размеры несколько меньше, однако порядок тотже. При этом распределение напряженности электрического поля по поверхности проволочного электрода неоднородно, причем максимум напряженности 

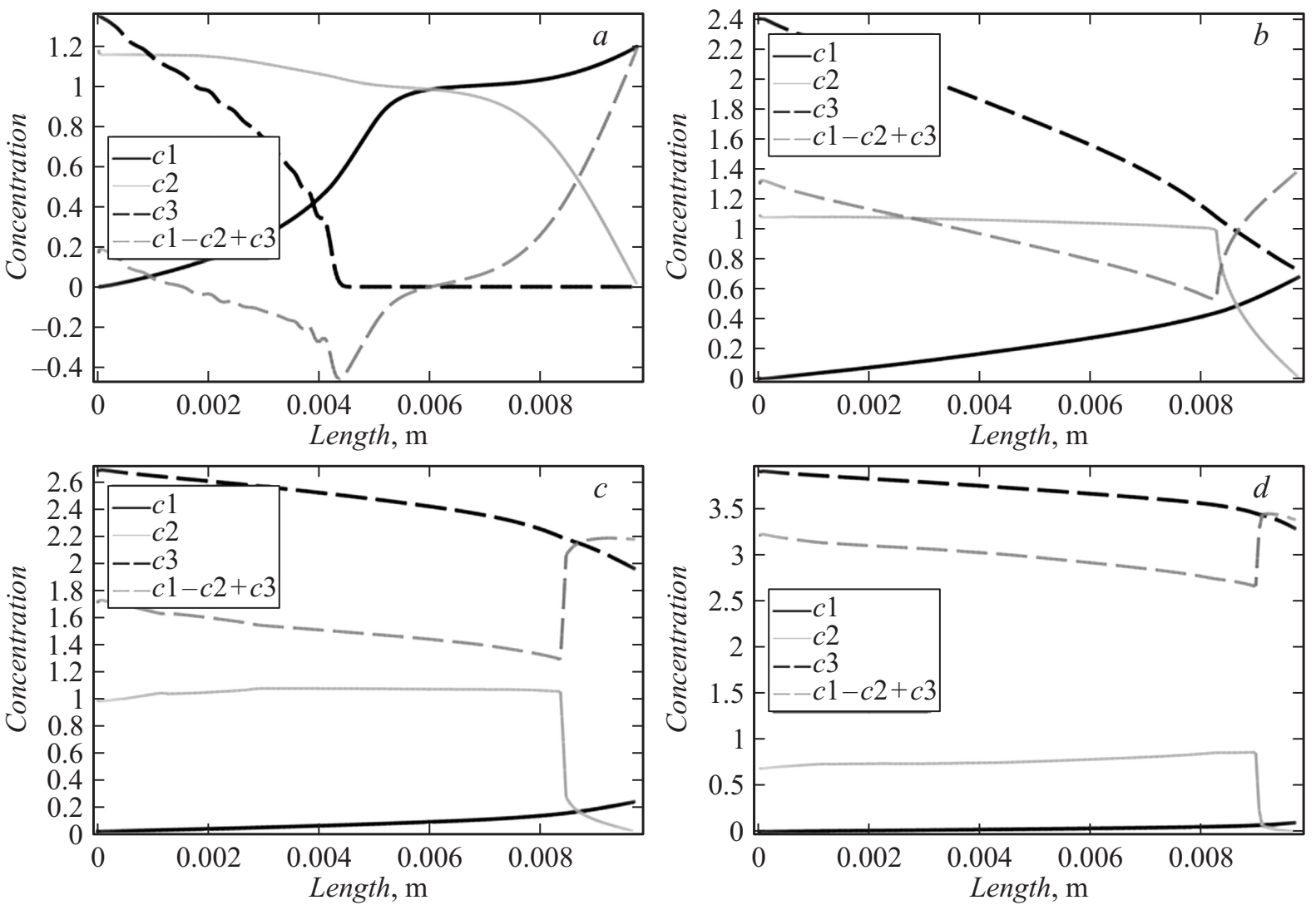

Рис. 3. Продольные распределения парциальных концентраций инжектированных положительных $(c 3)$, положительных $(c 1)$ и отрицательных $(c 2)$ диссоциированных ионов и их разности для различных напряжений: $a-2, b-2.5, c-10, d-17.5 \mathrm{kV}$.

соответствует нижней части электрода. Поэтому благодаря наличию заряженного гетерослоя у проволочного электрода при напряжении $1.5 \mathrm{kV}$ наблюдаются слабые течения, направленные вдоль поверхности электрода снизу вверх. У плоского электрода течений не наблюдается, а неравновесный слой сохраняет плоскую структуру, причем толщина слоя меньше. При напряжении $2 \mathrm{kV}$ безразмерная плотность тока инжекции $k$ с нижней части проволочного электрода становится больше 1 $(k>1)$, при этом, как и следовало ожидать, структура неравновесного приэлектродного слоя существенно изменяется. Сквозь кольцевой слой гетерозаряда с нижней части проволочного электрода в объем жидкости проникает струйка жидкости, содержащая ионы, инжектируемые на поверхности электрода. Эта струйка заряжена одноименно с электродом. Плотность заряда, проникающего в жидкость, очень мала, однако направление ЭГД течений изменяется на противоположное, а интенсивность течений существенно возрастает по сравнению с аномальным течением при напряжении $1.5 \mathrm{kV}$. Такое ЭГД течение является нормальным, а напряжение, соответствующее его возникновению, обычно называют пороговым. Скорость нормального течения на начальном этапе очень быстро возрастает, этот этап обычно называется этапом неразвитого ЭГД течения [1].
У плоского электрода структура неравновесного слоя остается практически неизменной.

С ростом напряжения до $2.5 \mathrm{kV}$ величина плотности безразмерного тока инжекции быстро возрастает, поэтому возрастает плотность объемного заряда в струе, а заряженная струя пересекает межэлектродный промежуток, упираясь в неравновесный приэлектродный слой у плоского электрода, нарушая его структуру. Судя по линиям тока, в жидкости реализуется замкнутое ячеистое ЭГД течение. Поскольку скорость ЭГД течения в десятки раз выше скорости движения ионов, то за время максвелловской релаксации, характеризующей диссоционнорекомбинационные процессы, приэлектродные слои проникают вглубь жидкости, образуя струйки. Этот процесс лучше виден на линейных продольных распределениях парциальных концентраций, приведенных на рис. 3.

Из рис. 3 видно, что распределения парциальных концентраций инжектированных ионов и концентрации ионов, формирующих объемный заряд при различных напряжениях, существенно различны. Так, при малых напряжениях (до $2 \mathrm{kV}$ ), когда безразмерный токовый параметр $k$ близок к 1 , у проволочного и плоского электродов имеются приэлектродные слои пониженной концентрации диссоциированных ионов, при этом инжектированные ионы проникают в жидкость не более чем до половины межэлектродного промежутка. При 
этом скорости ЭГД течений пока еще очень малы. Распределение концентрации ионов, формирующих объемный заряд, существенно отлично от распределения концентрации инжектированных ионов.

При незначительном повышении напряжения до $2.5 \mathrm{kV}$ структура приэлектродных слоев существенно изменяется. Это связано с возникновением нормальных ЭГД течений и их влиянием на структуру приэлектродных слоев. Видно, что инжектируемые положительные ионы проникают в объем жидкости, достигая противоэлектрода, приэлектродный слой дефицита положительных диссоциированных ионов преобразуется в струйку пониженной концентрации этих ионов, достигающей противоэлектрода. Распределение концентрации отрицательных ионов практически не изменяется: равновесное распределение по всему объему, за исключением неравновесного приэлектродного слоя пониженной концентрации. Повышенная концентрация инжектированных положительных ионов и дефицит положительных диссоциированных ионов сносятся течением в объем жидкости, образуя зеркально симметричные распределения вдоль струй. В связи с этим распределения концентрации ионов, формирующих распределение плотности объемного заряда, существенно отлично от распределения концентрации инжектированных ионов. Как видно из распределений рис. 3, распределение объемного заряда в начальной части струйки линейно спадает в направлении течения, так же как и распределение плотности инжектированных ионов, однако у плоского электрода наблюдается существенный рост. Этот рост определяется структурой неравновесного приэлектродного слоя отрицательных ионов.

По мере повышения напряжения безразмерный токовый параметр $k$ быстро возрастает. Можно сказать, что при малых напряжениях инжекция слабая, а при напряжении $10 \mathrm{kV}$ инжекция сильная. Как видно из распределений рис. 3, по мере роста безразмерного параметра $k$ (силы инжекции) влияние неравновесных приэлектродных слоев падает. При этом структура струйки инжектированных ионов становится все более однородной, а рекомбинационный эффект исчезает. Распределение объемного заряда вдоль струи при напряжении $20 \mathrm{kV}$ практически повторяет распределение концентрации инжектированных ионов.

\section{Результаты моделирования для жидкости с повышенной проводимостью $1 \mathrm{nS} / \mathrm{m}$}

Рассмотрим результаты моделирования в жидкости № 2 с более высокой низковольтной проводимостью, равной $1 \mathrm{nS} / \mathrm{m}$. На рис. 4, $a$ приведены распределения плотности объемного заряда в межэлектродном промежутке для напряжений $1.5,2,3,6,15,20 \mathrm{kV}$, a на серии рис. 4, $b$ - распределения модуля скорости ЭГД течений. В этой жидкости безразмерный токовый параметр $k$ становится равным единице при интенсивности инжекции на два порядка выше, чем в жидкости № 1. При относительно слабой инжекции инжектируемые ионы рекомбинируют в приэлектродном неравновесном слое, не проникая в объем жидкости. В таких условиях при напряжении $1.5 \mathrm{kV}$ у проволочного электрода реализуется слабое ЭГД течение, направленное на проволочный электрод, практически не влияющее на структуру неравновесного приэлектродного слоя, имеющего кольцевую структуру. Из рис. 4 видно, что при пороговом напряжении $2 \mathrm{kV}$ безразмерный параметр $P_{0}=100$, т. е. толщина приэлектродных слоев, рассчитанная в плоском приближении, на два порядка ниже, чем в жидкости № 1. В нормальном масштабе этот слой слабо различим. Поэтому на первых трех графиках показана увеличенная часть межэлектродного промежутка, позволяющая различить приэлектродный слой и тонкую струйку инжектированных ионов, проникающую от проволочного электрода в объем жидкости. При отсутствии инжекции $(1.5 \mathrm{kV})$, так же как и в предыдущем случае, у электродов формируются неравновесные диссоционно-рекомбинационные слои, заряженные противоположно электроду. У проволочного электрода слои имеют кольцевую структуру, у плоского электрода плоскую. Поперечные размеры приэлектродных слоев значительно меньше, чем для жидкости № 1. Слабые ЭГД течения имеют аномальное встречное направление и не влияют на приэлектродные слои. При напряжении $2 \mathrm{kV}(k=1)$ структура кольцевого приэлектродного слоя существенно изменяется: возникает нормальное ЭГД течение, направленное от проволочного электрода к плоскому. Это течение сносит инжектированные ионы с нижней части проволочного электрода в жидкость, формируя тонкую струйку инжектированных положительных ионов, быстро рекомбинирующих с отрицательными ионам жидкости. При напряжении $3 \mathrm{kV}$ под проволочным электродом формируется удлиненная струйка жидкости, заряженная одноименно с электродом.

Поскольку толщина рекомбинационного слоя меньше, чем у жидкости № 1, толщина заряженной струйки, образованной из приэлектродного слоя, также значительно меньше диаметра проволочного электрода. При напряжении $15 \mathrm{kV}$ струйка пересекает межэлектродный промежуток, а ее толщина еще более уменьшается. ЭГД течение переходит в развитой режим и приобретает выраженный двухячеистый характер.

На рис. 5 приведены линейные распределения парциальных концентраций ионов 1-, 2-, 3-го сортов, а также их разности (плотности объемного заряда) для напряжений 2, 3, 10, $20 \mathrm{kV}$. При напряжениях $2 \mathrm{kV}$ и ниже у обоих электродов наблюдаются тонкие (менее $1 \mathrm{~mm}$ ) неравновесные слои: недостатка положительных ионов сорта 1 у проволочного электрода и отрицательных сорта 2 у плоского электрода. Инжектированные ионы сорта 3 проникают в межэлектродный промежуток не далее глубины неравновесных приэлектродных слоев, рекомбинируя с отрицательными ионами 

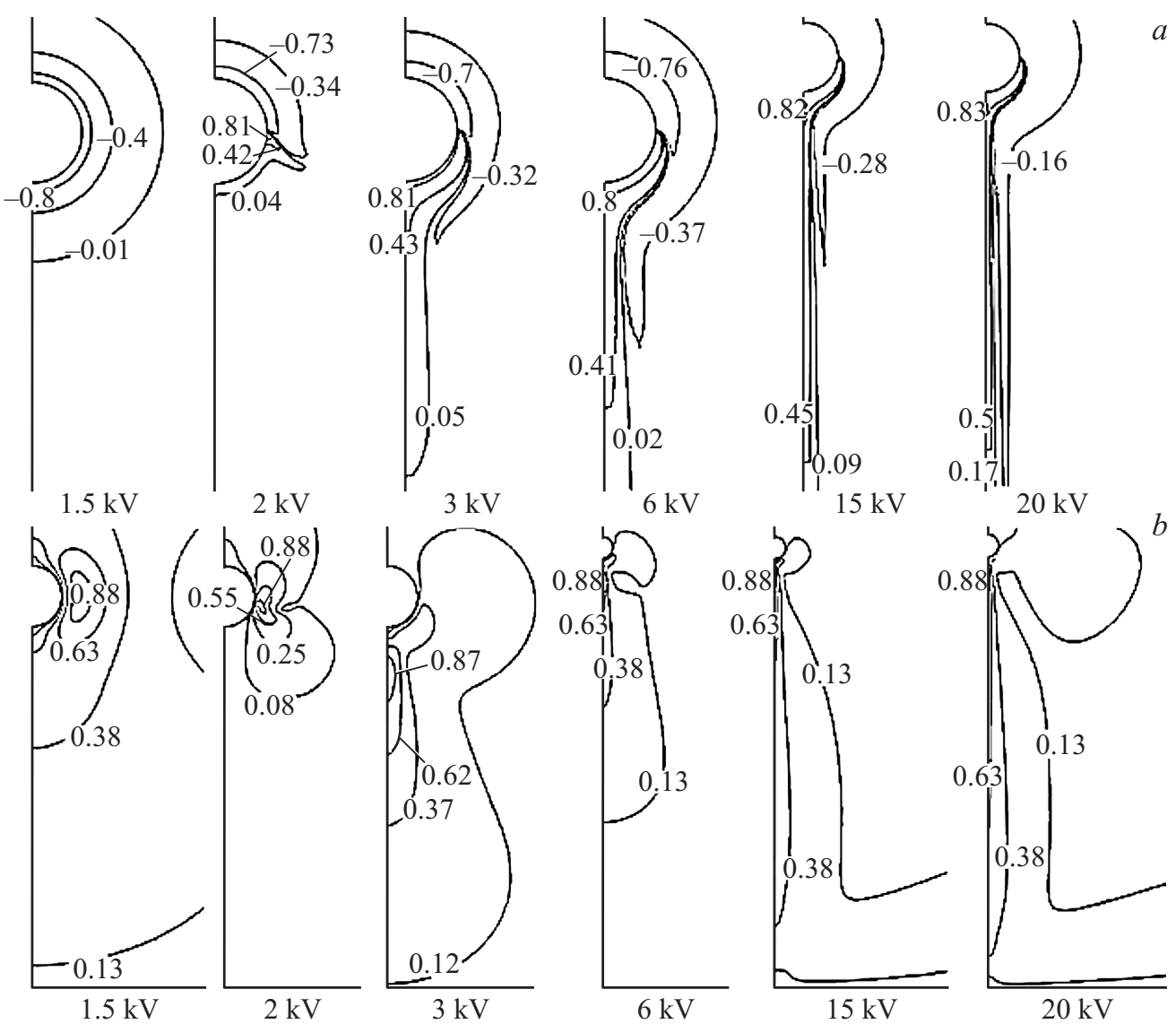

Рис. 4. $a-$ распределения объемного заряда в МЭП при различных напряжениях в жидкости № 2. $b-$ распределения скорости ЭГД течений при различных напряжениях в жидкости № 2.
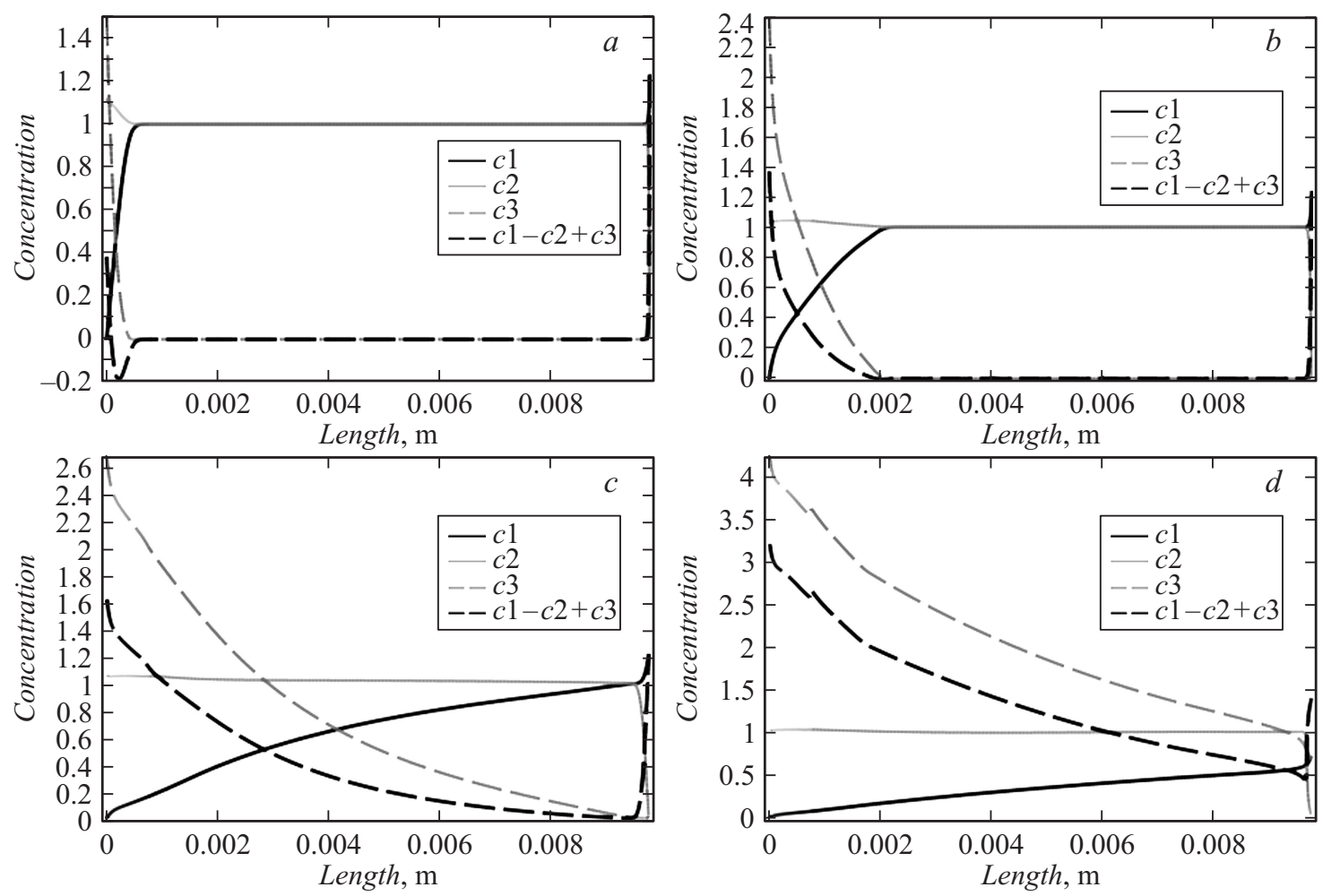

Рис. 5. Продольные распределения парциальных концентраций ионов 1-, 2- и 3-го сортов и парциальной концентрации ионов, формирующих объемный заряд (их разность) для напряжений: $a-2, b-3, c-10, d-20 \mathrm{kV}$. 
жидкости. Объемный заряд сосредоточен в пределах неравновесных слоев и имеет гетерозарядный характер. При напряжении $3 \mathrm{kV}$ структура неравновесного слоя у плоского электрода сохраняется, а у проволочного электрода изменяется. Как и ранее, из приэлектродного слоя формируется заряженная струйка ЭГД течения, которая сносит инжектированные ионы (c3) в объем жидкости. Эта же струйка сносит в объем жидкости и недостаток диссоциированных ионов сорта 1. За существенно уменьшенное время максвелловской релаксации, составляющее для жидкости № $2-0.01 \mathrm{~s}$, происходит рекомбинация инжектированных ионов $(c 3)$ с отрицательными ионами (c2), а также восстановление равновесной концентрации положительных ионов $(c 1)$ за счет диссоциации. Поэтому глубина проникновения струйки инжектированных ионов при напряжении $3 \mathrm{kV}$ невелика и возрастает до длины межэлектродного промежутка только при более высоком напряжении в $10 \mathrm{kV}$. В связи с этим значительно увеличен интервал напряжений перехода от режима неразвитого течения к режиму развитого ЭГД течения. При дальнейшем увеличении напряжения структура ЭГД течения не изменяется, увеличивается только интенсивность инжекции, т.е. величина безразмерного токового параметра $k$ и плотность заряда в струе. По мере перехода в режим сильной инжекции рекомбинационный эффект уменьшается, а распределение концентрации инжектированных ионов все менее отличается от распределения концентрации ионов, формирующих объемный заряд.

\section{Заключение}

Проведено компьютерное моделирование ЭДГ течений в системе электродов „провод над плоскостью“ на основе комплексного решения полной системы уравнений электрогидродинамики для случая трехионной жидкости. Получены решения для двух жидкостей с собственной равновесной проводимостью 10 и $1000 \mathrm{pS} / \mathrm{m}$. Зависимости функции инжекции от локального значения напряженности электрического поля на поверхности проволочного электрода заданы по формуле Шоттки и практически линейны.

Анализ результатов решения показал:

1. В диэлектрической жидкости с собственной проводимостью в диапазоне низких напряжений до $U_{\mathrm{thr}} \mathrm{y}$ каждого электрода формируются неравновесные слои, в пределах которых гибнут инжектированные ионы. При напряжении выше $U_{\text {thr }}$ у проволочного электрода наблюдаются прорыв приэлектродного рекомбинационного слоя и образование из него тонкой струйки инжектированных ионов. С увеличением напряжения реализуется два режима ЭГД течений: режим слабой инжекции: $k \sim 1$ и режим сильной инжекции: $k \gg 1$. В режиме слабой инжекции наблюдается существенная рекомбинация инжектированных ионов с противоионами собственной проводимости. В режиме сильной инжекции рекомбинационный эффект незначительный.

2. С увеличением собственной проводимости жидкости инжектируемый заряд более интенсивно рекомбинирует в неравновесном слое, поэтому для выполнения условия $k>1$ необходимо пропорционально увеличить интенсивность инжекции. С увеличением равновесной проводимости время максвелловской релаксации $\tau=\frac{\varepsilon \varepsilon_{0}}{\sigma_{0}}$ и толщина приэлектродного неравновесного слоя падают. Поэтому в жидкости с проводимостью $1000 \mathrm{pS} / \mathrm{m}$ приэлектродные слои значительно тоньше, соответственно тоньше и заряженные струйки ЭГД течений. Увеличивается также диапазон напряжений, при котором ЭГД течение выходит в развитой режим.

3. Результаты моделирования соответствуют результатам экспериментов, приведенных в $[1,8]$.

Компьютерное моделирование проведено с использованием вычислительных ресурсов „Вычислительного центра СПбГУ““ (http://cc.spbu.ru).

\section{Список литературы}

[1] Стишков Ю.К., Остапенко А.А. Электрогидродинамические течения в жидких диэлектриках // Л.: Изд-во Ленингр. ун-та, 1989. $174 \mathrm{c}$.

[2] Стишков Ю.К. // Докл. АН СССР. 1986. Т. 288. № 4.

[3] Traor'e Ph., Daaboul M., LoustCh. // J. Phys. D: Appl. Phys. 2010. Vol. 43. P. 8.

[4] Полянский В.А., Панкратьева И.Л. // Сб. докл. ХІ междунар. научной конф. „Современные проблемы электрофизики и электрогидродинамики жидкостей“. 2015. С. 219-223.

[5] Стишков Ю.К., Чирков В.А. // Сб. докл. ХІ Междунар. научной конф. „Современные проблемы электрофизики и электрогидродинамики“. 2015. С. 211-215.

[6] Жакин Д.И. // УФН. 2006. Т. 176. № 3. С. 289-310.

[7] Стишков Ю.К., Чирков В.А. // ЖТФ. 2012. Т. 82. Вып. 1. C. 3-13.

[8] СтишковЮ.К., Ситников А.А., Чирков В.А. // Сб. докл. XI Междунар. научной конф. „Современные проблемы электрофизики и электрогидродинамики“. 2015. С. 49-54. 